\title{
SIMULATION OF POLYCRYSTALLINE BISMUTH FILMS SEEBECK COEFFICIENT BASED ON EXPERIMENTAL TEXTURE IDENTIFICATION
}

\author{
Alexander S. Fedotov ${ }^{1}$, Vasiliy Shepelevich ${ }^{1}$, Sergey Poznyak ${ }^{2}$, Lyudmila Tsybulskaya ${ }^{2}$, \\ Alexander Mazanik ${ }^{1}$, Ivan Svito ${ }^{1}$, Sofia Gusakova ${ }^{1}$, Pawel Zukowski ${ }^{3}$, \\ Tomasz N. Koltunowicz ${ }^{3, *}$ \\ ${ }^{1}$ Belarusian State University, Minsk, Belarus \\ ${ }^{2}$ Research Institute for Physical Chemical Problems, Belarusian State University, Minsk, \\ Belarus \\ ${ }^{3}$ Lublin University of Technology, Lublin, Poland
}

\begin{abstract}
Seebeck coefficient values measured for textured polycrystalline Bi films have been compared with numerically computed ones. The proposed computation procedure requires crystallographic texture mapping and values of single-crystalline transport coefficients as input data. The comparison of computed and measured Seebeck coefficients allowed determining the factors affecting films' transport properties except of crystalline texture. Two particular cases are considered: when the film properties are affected only by texture and when they depend on texture and grain size simultaneously.
\end{abstract}

Keywords: Bismuth; Polycrystalline films; Crystal structure; Transport properties.

\section{Introduction}

It is common knowledge that properties of polycrystalline materials are determined by such factors as interface electronic states, mean free path limitations, and, in the case of materials with a pronounced anisotropy of single-crystalline properties, by texture type [1-3]. It is a challenge usually to distinguish these physical mechanisms, especially when crystallites are highly anisotropic and film shows a clearly defined texture.

Bismuth is known as semimetal possessing a significant anisotropy of transport properties in the single-crystalline form. It is a basic material for many of thermoelectrical applications $[4,5]$ that justifies a search for the ways of its Seebeck coefficient improvement $[6]$.

In the present report Seebeck coefficient of polycrystalline bismuth films with different grain sizes and crystallographic texture has been studied. We propose a technique for computation of polycrystalline film's Seebeck coefficient on the basis of solution of a partial differential equation system, considering each grain as a continuous medium with transport coefficients corresponding to the texture. Additional factors, except of crystalline texture, affecting the transport properties of polycrystalline Bi films are also considered.

\section{Preparation of samples and texture identification}

Two types of polycrystalline bismuth films were studied: (a) films prepared by the melt spinning (MS) and (b) ones obtained by the electrochemical deposition (ECD). Both synthesis techniques were well-developed and tested in our previous works $[7,8]$.

In the MS technique, melted $\mathrm{Bi}$ was spilled on the room-temperature surface of a rotating copper cylinder. Cooling rate of $\mathrm{Bi}$ reached appr. $10^{6} \mathrm{~K} / \mathrm{s}$ [9]. Thickness of the fabricated MS films was about $40 \mu \mathrm{m}$.

ECD synthesis was performed in the galvanostatic mode from an aqueous electrolyte, containing $0.174 \mathrm{M}$ bismuth perchloride dissolved in $3 \mathrm{M}$ perchloric acid. The

\footnotetext{
* Corresponding author e-mail:t.koltunowicz@pollub.pl, fax: +48-81-5384575, tel: +48-81-5384713
} 
electrodeposited $\mathrm{Bi}$ films were rinsed with distilled water and then picked off from the substrate. Thickness of the electrodeposited films was about $70 \mu \mathrm{m}$.

Several series of samples were fabricated by both methods under the same conditions. Five samples obtained by the MS and more than ten ECD films were investigated by scanning electron microscopy and demonstrated good reproducibility in their structure.

For experimental determination of Seebeck coefficient, a heater element was powered by an Agilent U3606B DC power source creating a temperature gradient about $1.5 \mathrm{~K}$ on the sample investigated. Temperatures on cold and hot ends of the sample were determined using platinum resistance thermometers connected to Agilent 34410A digital multimeters and providing an accuracy of temperature determination about $0.05 \mathrm{~K}$. Thermal EMF was measured by an Agilent 34411A multimeter with an accuracy of about $3.5 \mu \mathrm{V}$.

Samples for electrophysical measurements were cut from the center of each assynthesized film to avoid an influence of boundaries on the structure. Seebeck coefficients determined for different samples of each type fluctuate around the corresponding average values within $1 \%$.

A HZG-4M X-ray diffractometer ( $\mathrm{Cu} \mathrm{K \alpha}$ radiation) and a LEO 1455VP scanning electron microscope with an add-on for electron backscatter diffraction (EBSD) analysis were used for identification of the texture.

According to the SEM data, Bi grain sizes range from 5 to $15 \mu \mathrm{m}$ for the films fabricated by the MS and from 0.5 to $1.5 \mu \mathrm{m}$ for the ECD ones.

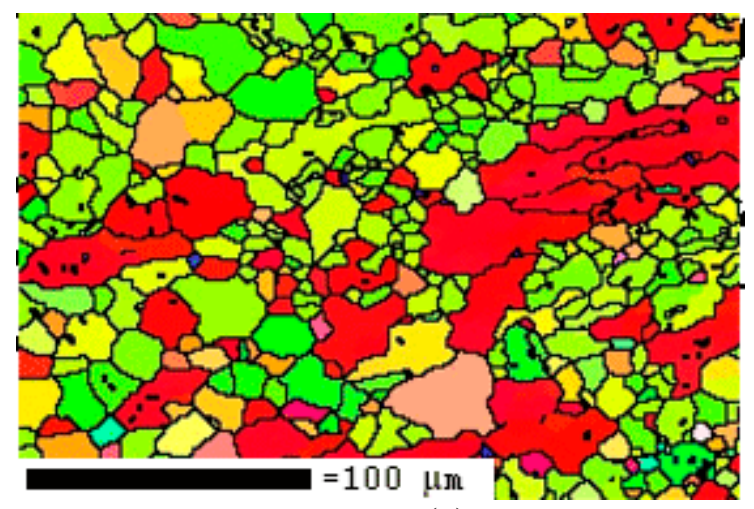

(a)

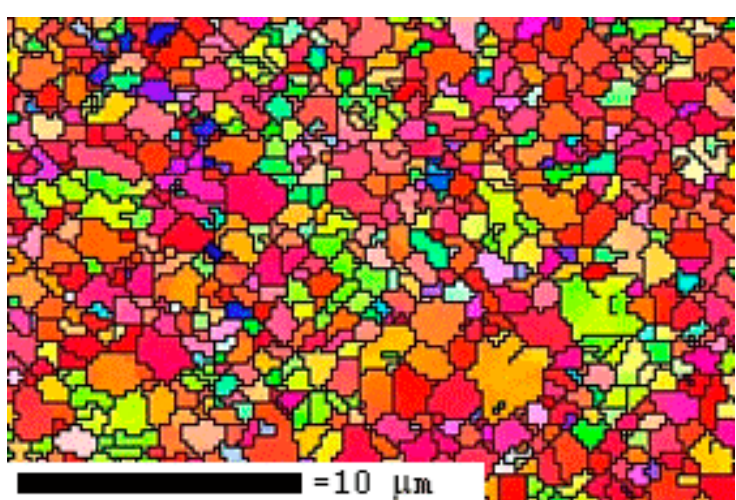

(b)

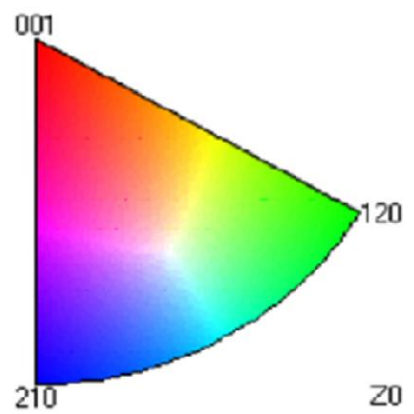

(c)

Fig. 1. EBSD texture mapping for particular grains (in-film plane) of the MS (a) and ECD films $(b)$ and the color legend $(c)$.

As follows from Fig. 1, two types of in-plane growth texture are observed: (001) and (012). XRD analysis (Fig. 2a) demonstrated only the presence of reflex corresponding to (012), but this divergence is well-known and related to the relaxation of the (001) line in bismuth [10]. Quantitative EBSD analysis showed that the films fabricated by the MS possess (001) and (012) textures for 39\% and 54\% of grains, respectively. The ECD films have (001) 
texture for $36 \%$ and (012) texture for $57 \%$ of the grains. Relevant crystallographic planes are shown in Fig. $2 b$.

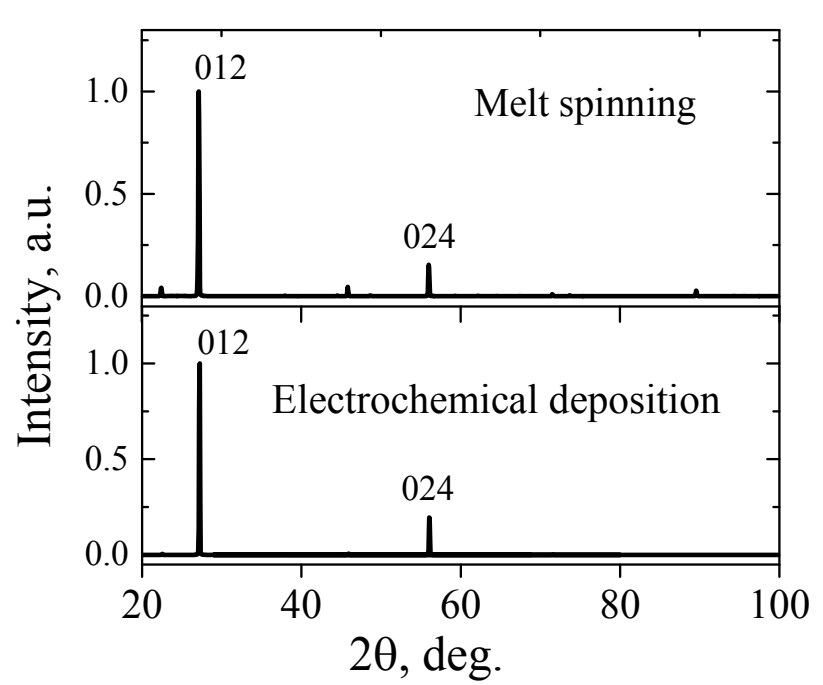

(a)

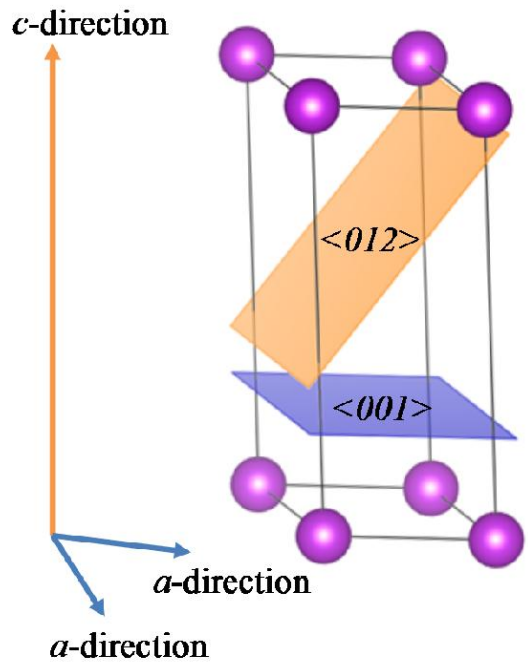

(b)

Fig. 2. XRD patterns $(a)$ and the corresponding crystallographic planes $(b)$ in Bi lattice.

\section{Mathematical model and Seebeck coefficient computation}

We can use the following equation system [11] to describe the coupled processes of heat and charge transfer:

$$
\left\{\begin{array}{l}
\vec{J}=-\sigma(\nabla V+S \nabla T) \\
\rho C_{\mathrm{p}} \frac{\partial T}{\partial t}=\nabla \cdot(k \nabla T+S T \cdot \vec{J})+\vec{J} \nabla V
\end{array},\right.
$$

where $\vec{J}$ is the current density, $T$ - the temperature, $V$ - the electric potential, $S$ - the Seebeck coefficient, $\sigma$ - the electrical conductivity, $k-$ the thermal conductivity, $C_{\mathrm{p}}-$ the heat capacity, $\rho$ - the density, and $t$ - the time.

We have set the computational domain consisted of 48 subdomains (according to the SEM data, the MS films possess a column-like structure). Each subdomain is $15 \times 15 \times 40 \mu^{3}$ parallelepiped (Fig. 3), corresponding to the measured foil thickness $(40 \mu \mathrm{m})$ and average lateral grain size $(15 \mu \mathrm{m})$. The whole domain size is $120 \times 90 \times 40 \mu \mathrm{m}^{3}$.

For computation of Seebeck coefficient, boundary conditions were set to correspond to the open circuit regime at a small temperature gradient between two opposite faces, while the rest boundary faces were considered as thermally insulated:

$$
\left\{\begin{array}{l}
T=294, x=0,(y, z) \in \Gamma \\
T=293, x=120,(y, z) \in \Gamma \\
\vec{n} \cdot \vec{J}=0, x \in \Gamma \\
\vec{n} \cdot(-k \nabla T)=0,0<x<120,(y, z) \in \Gamma
\end{array},\right.
$$

where $\Gamma$ is the boundary of the domain and $\vec{n}$ is the unit vector normal to it. 
For one of the faces, between which the temperature gradient was set, the potential was considered as equal to zero. The value of thermo-EMF was obtained by averaging the voltage through entire face with non-zero-voltage condition.

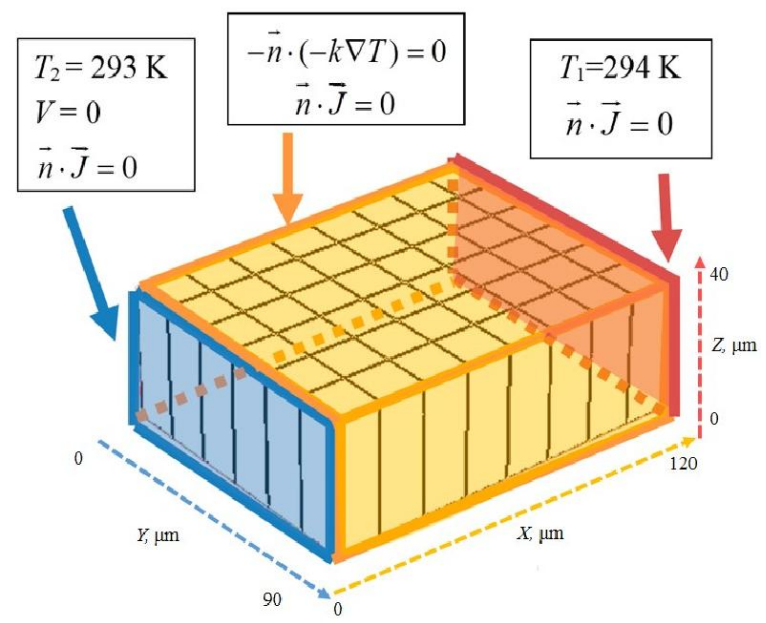

Fig. 3. Boundary conditions over the computational domain.

For quantitative predictions of the electrical conductivity, additional initial-boundary value problems were solved on the same computational domain:

$$
\left\{\begin{array}{l}
T=293,(x, y, z) \in \Gamma \\
\vec{n} \cdot \vec{J}=1 \cdot 10^{-5}, x=0,(y, z) \in \Gamma \\
\vec{n} \cdot \vec{J}=-1 \cdot 10^{-5}, x=120,(y, z) \in \Gamma \\
\vec{n} \cdot \vec{J}=0,0<x<120,(y, z) \in \Gamma
\end{array} .\right.
$$

The voltage drop across the rectangular sample was obtained by averaging potential distributions on the both ends of the sample (for $x=0$ and $x=120$ boundaries) and subtracting one from another. The conductivity of the sample was determined as a ratio of the current density to the voltage drop, multiplied by the sample length.

All the simulations were performed for stationary cases using the finite element method. Mesh consisted of $5 \cdot 10^{5}$ tetrahedral elements. Conventional PARDISO 5.0 solver was used with pivoting perturbation order of $10^{-8}[12,13]$.

Transport coefficients of single crystal, such as conductivity, Seebeck coefficient and others, are tensors [14]. To take into account the crystalline texture, it is necessary to transform the tensors using the rotation matrix [15]. However, some geometrical relations could simplify the procedure of transport properties assignment to the simulation domain. Bismuth lattice cell is suitable to be described in the hexagonal coordinate system [10, 14]. Therefore it is common to distinguish two types of crystallographic directions with their own transport coefficients. The first one coincides with $\mathrm{C} 3$ symmetry axis of the crystal cell and is called $c$ direction, whereas the directions lying in the plane normal to the $\mathrm{C} 3$ axis are called $a$ directions (Fig. 2b). If the (001) plane is parallel to the film surface, it leads to $a$ direction coefficients for film in-plane transport (Fig. 1b). In the case of the (012) plane parallel to the surface, transport processes occur with the values of transport coefficients ranging between a lower limit (values for $a$ direction ) and an upper limit $C_{\text {up, which can be approximated as }}$

$$
C_{u p}=C_{a}+\left(C_{c}-C_{a}\right) \sin (\Theta)
$$


where $\theta$ is the angle between the (001) and (012) planes, $C_{a}$ and $C_{c}-$ the transport coefficients for corresponding directions taken from [14].

For the MS films, we excluded from the consideration $7 \%$ of the grains, which do not show (001) or (012) dominating textures. Then, we assumed that every subdomain has the probability to possess transport coefficients of $a$ direction equal to $71 \%$. The rest $29 \%$ of grains oriented with the (012) plane parallel to the sample surface have equal probabilities to possess transport coefficients corresponding to $a$ direction or coefficients as given in (4) (bimodal distribution with equal probabilities). Similar analysis was also performed for the ECD films taking into account peculiarities of their texture.

Fig. 4 shows the dependence of Seebeck coefficient and electrical conductivity on the volume percentage, $x$, of the grains oriented with $c$ direction parallel to the temperature gradient, whereas the rest of the grains are oriented with $a$ direction parallel to the temperature gradient. A linear dependence of Seebeck coefficient on the fraction of grains oriented with $c$ direction is obtained as a result of the calculations.

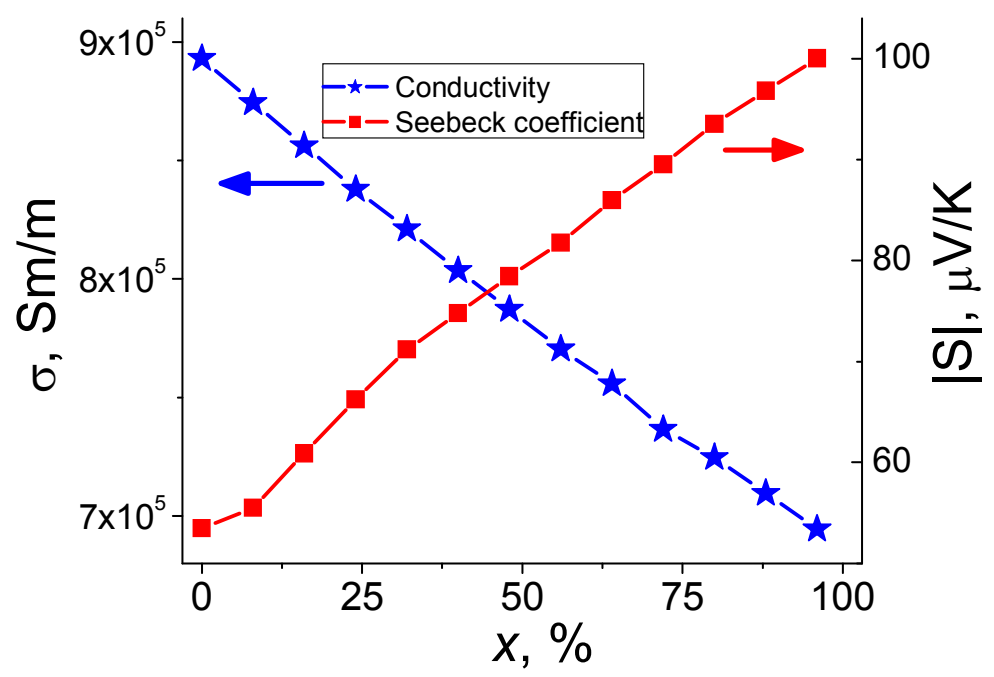

Fig. 4. Calculated dependences of Seebeck coefficient and electrical conductivity for ideal bitextured film on the volume percentage of the grains oriented with $c$ direction parallel to the temperature gradient.

The performed calculation for the MS films returned the value of Seebeck coefficient equal to $62 \mu \mathrm{V} / \mathrm{K}$, whereas the experimentally measured value is $63 \mu \mathrm{V} / \mathrm{K}$. For the ECD films the divergence is rather large: $66 \mu \mathrm{V} / \mathrm{K}$ according to the calculations and $75 \mu \mathrm{V} / \mathrm{K}$ in the experiment.

As mentioned above, the grain size for the ECD films ranges from 0.5 to $1.5 \mu \mathrm{m}$. The mean free path of charge carriers in bismuth at $300 \mathrm{~K}$ is about $250 \mathrm{~nm}$ [16]. It is reasonable to suggest that the limitation of charge carrier mean free path by the scattering on grain boundaries results in modification of the transport properties. Therefore, it is improperly to use experimental data related to large-sized single-crystals for defining the transport coefficients of the fine-grained ECD films.

Mobilities of electrons $\mu_{\mathrm{n}}$ and holes $\mu_{\mathrm{p}}$ can be calculated using Seebeck coefficient and electrical conductivity:

$$
\sigma=e \cdot\left(n \mu_{\mathrm{n}}+p \mu_{\mathrm{p}}\right)
$$




$$
S=\frac{S_{\mathrm{n}} n \mu_{\mathrm{n}}+S_{\mathrm{p}} p \mu_{\mathrm{p}}}{n \mu_{\mathrm{n}}+p \mu_{\mathrm{p}}} .
$$

Here the concentrations of electrons, $n$, and holes, $p$, are independent on texture and can be taken from [8]. Partial Seebeck coefficients $S_{\mathrm{n}}$ and $S_{\mathrm{p}}$ can be calculated using the Lax model [16].

Results of the computation demonstrate (Fig. 5) that the mobility of electrons is almost independent on the texture, while the mobility of holes decreases appreciably with $x$. This fact can be explained taking into account that $T$-holes pocket is prolate in $k$-space along the $c$ direction, while three pockets of $L$-electrons are distributed symmetrically [17]. Such form of the $T$-holes pocket leads to a higher effective mass of holes in the direction of prolongation as compared with that in the other two directions and, consequently, to the reduction of holes mobility in $c$ direction.

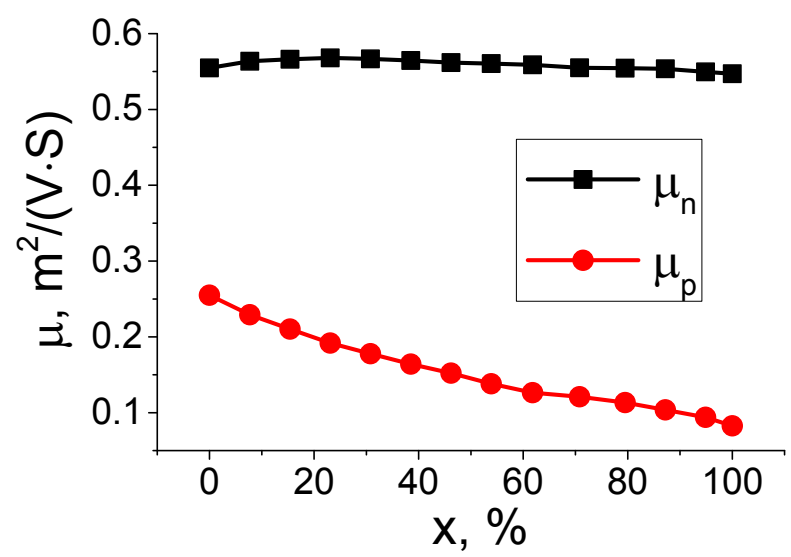

Fig. 5. Calculated mobilities of electrons (squares) and holes (circles) for bi-textured film depending on the volume percentage of grains oriented with $c$ crystallographic direction parallel to the temperature gradient.

\section{Conclusions}

Two types of polycrystalline bismuth films were examined to highlight factors affecting their transport properties depending on crystalline texture.

The proposed numerical technique predicts successfully Seebeck coefficient for the samples synthesized by the melt spinning and possessing a clearly defined texture. The results of calculation coincide with the experimental data with a high accuracy (about $1 \%$ ). For the samples synthesized by the electrochemical deposition, discrepancy between the calculated and experimental values of Seebeck coefficient reaches $15 \%$, indicating the presence of additional factors affecting Seebeck coefficient in such films. The last is in agreement with SEM analysis data showing that grain size for the ECD samples is of the order of charge carriers' mean free path that produces an additional impact on the transport properties.

The computation technique described can be used for determining Seebeck coefficient of anisotropic polycrystalline (or just composite-like) materials and has to be applicable to the films with grain sizes significantly larger than mean free path of charge carriers.

\section{References}

[1] U.F. Kocks, C.N. Tomé, R. Wenk, Preferred Orientations in Polycrystals and their Effect on Materials Properties, Cambridge University Press, (2000).

[2] S. Datta, Electronic Transport in Mesoscopic Systems, Cambridge University Press, (1995). doi:http://dx.doi.org/10.1017/CBO9780511805776. 
[3] M. Lundstrom, C. Jeong, Near-Equilibrium Transport. Fundamentals and Applications, World Scientific Publishing, (2013). doi:http://dx.doi.org/10.1142/7975.

[4] D.M. Rowe, Thermoelectrics Handbook: Macro to Nano, CRC Press, (2005). doi:http://dx.doi.org/10.1201/9781420038903.

[5] H.J. Goldsmid, Bismuth Telluride and Its Alloys as Materials for Thermoelectric Generation, Materials 7 (2014) 2577-2592. doi:http://dx.doi.org/10.3390/ma7042577

[6] H.J. Goldsmid, Introduction to Thermoelectricity, Springer, Berlin, (2010). doi:http://dx.doi.org/10.1007/978-3-642-00716-3.

[7] A.S. Fedotov, S.K. Poznyak, L.S. Tsybulskaya, V.G. Shepelevich, I.A. Svito, A. Saad, A. Mazanik, A.K. Fedotov, Polycrystalline bismuth films: correlation between grain structure and electron transport, Phys. Status Solidi B, 252 (9) (2015) 2000-2005. doi:http://dx.doi.org/10.1002/pssb.201552051.

[8] A.S. Fedotov, S.K. Poznyak, L.S. Tsybulskaya, I.A. Svito, V.G. Shepelevich, A. V. Mazanik, A.K. Fedotov, T. V. Gaevskaya, Synthesis, structure and electrophysical properties of polycrystalline bismuth films, Proceedings of International Conference Sviridov Readings 2015, Minsk. http://elib.bsu.by/handle/123456789/118049.

[9] W.J. Boettinger, J H. Perepezko, J.H. Liebermann, Rapidly Solidified Alloys: Processes, Structures, Properties, Applications, Marcel Dekker, Inc, New York, (1993).

[10] P. Cucka, C.S. Barrett, The crystal structure of $\mathrm{Bi}$ and of solid solutions of $\mathrm{Pb}, \mathrm{Sn}, \mathrm{Sb}$ and $\mathrm{Te}$ in $\mathrm{Bi}$, Acta Crystallogr, 15 (9) (1962) 865-872. doi:http://dx.doi.org/10.1107/s0365110x62002297.

[11] L.D. Landau, E.M. Lifshitz, Electrodynamics of Continuous Media Edition, Butterworth Heinemann, Oxford, (1984). doi:http://dx.doi.org/10.1016/B978-0-08030275-1.50024-2.

[12] O. Schenk, M. Bollhöfer, and R. Römer, On large-scale diagonalization techniques for the Anderson model of localization, SIAM Review 50 (2008) 91-112. doi:http://dx.doi.org/10.1137/070707002.

[13] O. Schenk, A. Wächter, M. Hagemann, Matching-based Preprocessing Algorithms to the Solution of Saddle-Point Problems in Large-Scale Nonconvex Interior-Point Optimization, Comput Optim Appl 36 (2-3) (2007) 321-341. doi:http://dx.doi.org/10.1007/s10589-006-9003-y.

[14] B.S. Chandrasekhar, The seebeck coefficient of bismuth single crystals, J Phys Chem Solids 11 (1959) 268-273. doi:http://dx.doi.org/10.1016/0022-3697(59)90225-2.

[15] O. Engler, V. Randle, Introduction to Texture Analysis: Macrotexture, Microtexture, and Orientation Mapping, CRC Press, (2009). doi:http://dx.doi.org/10.1201/9781420063660.

[16] D. Nakamura, M. Murata, H. Yamamoto, Y. Hasegawa, T. Komine, Thermoelectric properties for single crystal bismuth nanowires using a mean free path limitation model, J Appl Phys 110 (2011) 053702. doi:http://dx.doi.org/10.1063/1.3630014.

[17] Z. Zhu, B. Fauque, Y. Fuseya, K. Behnia, Angle-resolved Landau spectrum of electrons and holes in bismuth, Phys Rev $\underline{B} \quad \underline{84} \quad$ (2011) 115137. doi:http://dx.doi.org/10.1103/physrevb.84.115137. 